\title{
Safety Impacts of Push-Button and Countdown Timer on Nonmotorized Traffic at Intersections
}

\author{
Bei Zhou, ${ }^{1}$ Arash M. Roshandeh, ${ }^{2}$ and Shengrui Zhang ${ }^{1}$ \\ ${ }^{1}$ School of Highway, Chang'an University, Xian 710064, China \\ ${ }^{2}$ School of Civil Engineering, Purdue University, West Lafayette, IN 47907, USA \\ Correspondence should be addressed to Bei Zhou; bzhou2007@gmail.com
}

Received 14 July 2014; Accepted 29 August 2014; Published 25 September 2014

Academic Editor: Wuhong Wang

Copyright (C) 2014 Bei Zhou et al. This is an open access article distributed under the Creative Commons Attribution License, which permits unrestricted use, distribution, and reproduction in any medium, provided the original work is properly cited.

\begin{abstract}
This paper applies the random parameters negative binominal model to investigate safety impacts of push-button and countdown timer on pedestrians and cyclists at urban intersections. To account for possible unobserved heterogeneity which could vary from one intersection to another, random parameters model is introduced. A simulation-based maximum likelihood method using Halton draws is applied to estimate the maximum likelihood of random parameters in the model. Dataset containing pedestrians' and cyclists' crash data of 1,001 intersections from Chicago is utilized to establish the statistical relationship between crash frequencies and potential impact factors. LIMDEP (Version 9.0) statistical package is utilized for modeling. The parameter estimation results indicate that existence of push-button and countdown timer could significantly reduce crash frequencies of pedestrians and cyclists at intersections. Increasing number of through traffic lanes, left turn lanes, and ratio of major direction AADT to minor direction AADT, tend to increase crash frequencies. Annual average daily left turn traffic has a negative impact on pedestrians' safety, but its impact on cyclists' crash frequency is statistically insignificant at $90 \%$ confidence level. The results of current study could provide important insights for nonmotorized traffic safety improvement projects in both planning and operational levels.
\end{abstract}

\section{Introduction}

Nonmotorized traffic plays an important role in urban transportation system. The major types of nonmotorized traffic refer to pedestrians and cyclists. As traffic congestion and air pollution become increasingly serious in many cities, nonmotorized traffic has drawn much more attention over the last decades. A number of metropolitan planning agencies have proposed transportation demand management strategies to encourage nonmotorized traffic mode utilization as a countermeasure to congestion and environmental problems. In order to promote the development of nonmotorized traffic, it is crucial to conduct in-depth study with regard to pedestrian and cyclist safety issues.

Urban intersections have received lots of attention in terms of their signal's timing optimization to minimize vehicles' delay only $[1,2]$ or simultaneously minimizing delays of both vehicles and pedestrians [3]. Compared to vehicles, pedestrians and cyclists are much more vulnerable in road crashes. Pedestrians comprise more than $22 \%$ of the 1.24 million people killed in traffic accidents [4]. That is, more than 270,000 pedestrians lose their lives on the roads every year globally. According to National Highway Traffic Safety Administration [5], 677 cyclists were killed and additional 48,000 were injured in traffic crashes in 2011 in US. Some researchers [6] compared fatality rates per mile of travel by different modes in the US. Based on their work, pedestrians are 23 times more likely to get killed in crashes than car occupants, and cyclists are 12 times more likely. In order to reduce the high risk of pedestrians' and cyclists' fatalities/injuries, researchers have studied various factors which might contribute to nonmotorized crash rates and injury severity. These factors include pedestrians'/cyclists' characteristics, vehicle driver characteristics, vehicle attributes, roadway features, environmental factors, and crash characteristics [7] which can be modeled for both through traffic movements and also turning movements at intersections [8]. Compared to other methods (developing new leg form impactors, increasing 
numbers of law enforcement officers, or enhancing safety education), installing push-button and countdown timer at intersections is a relatively economic approach to improve the safety of pedestrians and cyclists. Nevertheless, only a handful of papers paid close attention to impacts of push-button and countdown timer on nonmotorized traffic safety.

The present study applied the random parameters negative binominal model to explicitly assess impacts of pushbutton and countdown timer on pedestrians' and cyclists' safety at intersections within an urban network. Dataset including traffic accident information from 1,001 intersections of Chicago in 2010 was utilized for modeling purpose. As an extension of Poisson model, negative binominal model overcomes the overdispersion in data. Additionally, in order to interpret possible heterogeneity which could vary from one intersection to another, random parameters model was introduced.

The rest of this paper is organized as follows. Next section discusses relevant previous research regarding nonmotorized safety analysis; details about methodology and modeling procedures are presented in methodology section; empirical setting part describes dataset used in the current study; estimation results summarize modeling results and impacts of various factors on pedestrians' and cyclists' safety; the final section provides summary and conclusions.

\section{Related Work}

Nonmotorized traffic safety has drawn lots of attentions from both developed and developing countries in last decades. Researchers have conducted various studies to evaluate the possible factors affecting safety of pedestrians and cyclists. A mixed generalized ordered response model for examining pedestrians' and cyclists' injury severity level was proposed [7]. The study suggested that most important factors contributing to the nonmotorized injury severity are ages of individual, speed limits, locations, and time of day. Bayesian spatial framework was utilized to investigate effects of spatial correlation on pedestrian and cyclist crashes [9]. Significant differences were found between the predictor sets for pedestrian and cyclist crashes. In addition, Bayesian models with spatial correlation outperformed models that did not consider spatial correlation among TAZs. A logistic regression model was performed to analyze odds of pedestrians crossing intersections safely [10]. It was revealed that the most significant risk was the time gap, which could be easily misjudged from the speed of oncoming vehicles. In order to prevent lower extremity injuries of pedestrians in car collisions, LS-DYNA software was used to investigate the collision mechanism between a GMT bumper and the leg form impactor model [11]. The study suggested developing special leg form impactors for different countries with different average person height. A methodology was developed to quantify pedestrian safety effects related to existing site characteristics on urban and suburban arterials [12]. The study revealed that vehicle-pedestrian crash frequency was highest when the ratio of minor road AADT to major road AADT was the highest. And intersections with more lanes had highest vehicle-pedestrian crashes. In 2011, Hamann and Peek-Asa
[13] conducted a site-control study of 147 bicycle crash sites in Iowa. They examined crash risk by on-road bicycle facility present and by facility type. It was concluded that bicyclespecific pavement markings and signage could reduce the number of cyclist-vehicle crashes and related injuries and fatalities. Moore et al. [14] developed a mixed logit model (MXL) to analyze cyclist injury severity resulting from motor vehicle crashes. Based on this study, driver recognition of cyclists is a serious problem which could increase both frequency of bicycle-vehicle crashes and the degree of injury severity. Agbelie and Roshandeh [15] applied a randomparameters negative binomial model to investigate impacts of the signal related factors on crash frequency. Results showed that increasing the number of signal phases and traffic volume at intersection would increase the crash frequency in all intersections, whereas increasing the number of approach lanes and the maximum green time have an ambiguous effect: on one hand, increasing the crash frequency at some intersections and on the other hand, decreasing the crash frequency at some other intersections. Ibrahim et al. [16] conducted a study in Malaysia to investigate impacts of countdown timer on drivers' behavior. They found that intersections with countdown timer have significant effect on queue discharge headway.

Although lots of studies have been conducted with regard to nonmotorized traffic safety problems, very few of them considered presence of push-button and countdown timer at intersections in their proposed models. The major contribution of the current study is to specifically evaluate safety impacts of push-button and countdown timers on pedestrian and cyclist safety at intersections. Compared to abovementioned work, the proposed random parameters negative binominal model takes account of not only major and minor road AADTs, left turn AADT, number of left turn lanes, and number of through lanes, but also presence of push-button and countdown timer devices at intersections. The finding of current study could provide valuable guidance for decision-making of nonmotorized traffic safety improvement at intersections.

\section{Methodology}

3.1. Model Description. There are different approaches such as lognormal and loglinear methods for statistical modeling of crashes. When data distribution is skewed, lognormal regression modeling could be used [17]. This is a distribution where random variable $x$ has mean $\mu$ and variance $\sigma^{2}$. For lognormal distribution, the density function can be written as

$$
f(x)=\frac{\operatorname{EXP}\left(-[\ln (x)-\mu]^{2} / 2 \sigma^{2}\right)}{x(2 \pi)^{1 / 2} \sigma} ; \quad x>0,
$$

where $\mu$ and $\sigma$ are location and scale parameters of the lognormal distribution [18]. The mean and variance of the lognormal distribution are $\operatorname{EXP}\left(\mu+\sigma^{2} / 2\right)$ and $\operatorname{EXP}\left[2\left(\mu+\sigma^{2}\right)\right]$ $-\operatorname{EXP}\left[2 \mu+\sigma^{2}\right]$, respectively.

In lognormal distribution, the assumption is that the natural log of the crash frequency has a normal distribution 
with mean $\mu$ and variance $\sigma^{2}$. This model is especially used when the mean is relatively large. Compared to lognormal regression models, loglinear regression approaches have extensively been applied for crash modeling purposes. Poisson and negative binomial are two main types of loglinear models. In a Poisson regression model, the probability of occurring $y_{i}$ crashes at intersection $i$ during a given time interval, $P\left(y_{i}\right)$, is given by

$$
P\left(y_{i}\right)=\frac{\operatorname{EXP}\left(-\lambda_{i}\right) \lambda_{i}^{y_{i}}}{y_{i} !}
$$

where $\lambda_{i}$ is the expected number of crashes at intersection $i$ per period, $E\left[y_{i}\right]$. Poisson regression models are estimated by determining $\lambda_{i}$ (Poisson parameter) as a function of explanatory variables. The relationship between explanatory variables and the Poisson parameter is the loglinear model as

$$
\lambda_{i}=\operatorname{EXP}\left(\beta X_{i}\right),
$$

where $X_{i}$ is a vector of explanatory variables and $\beta$ is a vector of estimable parameters.

Although the Poisson model has been used in many crash-frequency analyses, it does not handle over- and underdispersion and can produce biased results [19]. In other words, Poisson distribution restricts the mean and variance to be equal, where, in some cases, the analysis could not produce correct solutions. If the mean and variance are not equal, the data is said to be either underdispersed (mean greater than variance) or overdispersed (mean less than variance). A number of studies already concluded that traffic accident data are generally significantly overdispersed. Hence, applying Poisson model could result in incorrect estimation.

Another loglinear regression model is negative binomial model. It is an extension of the Poisson model and can overcome overdispersion in the data. Unlike Poisson distribution where it assumes the mean and variance are equal, the negative binomial distribution does not hold this assumption. The negative binomial model for each observation $i$ can be written as

$$
\lambda_{i}=\operatorname{EXP}\left(\beta X_{i}+\varepsilon_{i}\right),
$$

where $\lambda_{i}$ is expected number of crashes at intersection $i, \beta$ is a vector of estimable parameters, $X_{i}$ is a vector of explanatory variables, and $\operatorname{EXP}\left(\varepsilon_{i}\right)$ is a gamma-distributed error term with mean and variance of 1 and $\alpha^{2}$, respectively. It allows the variance to differ from the mean as

$$
\operatorname{VAR}\left[y_{i}\right]=E\left[y_{i}\right]+\alpha E\left[y_{i}\right]^{2}
$$

The parameter $\alpha$ is referred to as overdispersion parameter. The negative binomial model utilizes a distribution function as shown in

$$
P\left(y_{i}\right)=\frac{\Gamma\left((1 / \alpha)+y_{i}\right)}{\Gamma(1 / \alpha) y_{i} !}\left[\frac{1 / \alpha}{(1 / \alpha)+\lambda_{i}}\right]^{1 / \alpha}\left[\frac{\lambda_{i}}{(1 / \alpha)+\lambda_{i}}\right]^{y_{i}},
$$

where $\Gamma$ is a gamma function, $y_{i}$ is crashes at intersection $i, \alpha$ is overdispersion parameter, and $\lambda_{i}$ is expected crashes at intersection $i$. It needs to be mentioned that the negative binomial model is the most commonly used approach for crash frequency modeling.

In order to account for possible heterogeneity which could vary from one intersection to another, random parameters model can be introduced. To develop a randomparameters model that accounts for possible unobserved heterogeneity across intersections, the individual estimable parameters are written as [20]

$$
\beta_{i}=\beta+\omega_{i}
$$

where $\omega_{i}$ is a randomly distributed term for each signalized intersection $i$ and it can take on a wide variety of distributions such as Weibull, Erlang, logistic, lognormal, and normal. In the current study, all selected random parameters are normally distributed.

3.2. Model Evaluation. Model evaluation involves two steps. The first step is to evaluate the significance of variables included in the proposed model. Estimated coefficients for each explanatory variable should be statistically significant. The current study applies $t$-test to examine the significance of coefficient with $90 \%$ confidence interval. Additionally, it is necessary to use goodness-of-fit measures to evaluate if the proposed model has sufficient explanatory and predictive power. The present study adopts ratio of log-likelihood index as goodness-of-fit measures, which could be calculated by the following equation:

$$
\rho^{2}=1-\frac{l(\beta)}{l(0)}
$$

where $l(\beta)$ refers to the log-likelihood value at convergence and $l(0)$ is log-likelihood value with constant only. As suggested by Ben-Akiva and Lerman [21], $\rho^{2}$ could be used to test if the model has sufficient explanatory and predictive power.

A simulation-based maximum likelihood method using Halton draws is used to estimate maximum likelihood of the random-parameters in the model. Halton draws has been shown to provide a more efficient distribution of draws for numerical integration than purely random draws [20]. In order to assess the impacts of one unit change of each variable on either pedestrians' or cyclists' crash frequency, marginal effects are computed [21]. LIMDEP (Version 9.0) statistical package is utilized for modeling purposes [20].

\section{Empirical Setting}

The dataset available for this study includes crash data from 1,001 intersections from Chicago, collected in 2010. Data consists of pedestrians' and cyclists' crash frequencies, average annual daily left turn traffic, average annual daily traffic for major and minor approaches, number of left turn lanes, number of through traffic lanes, and availability of push-button and countdown timer. All of these observations have been collected for each intersection. Table 1 summarizes description of each variable and associated mean and standard deviation values. 
TABLE 1: Descriptive statistics of crash-related variables.

\begin{tabular}{|c|c|c|}
\hline Variables & Mean & Std. deviation \\
\hline $\begin{array}{l}\text { Average annual pedestrians' crash } \\
\text { frequency at each intersection } \\
\text { (crashes/intersection) }\end{array}$ & 4.98 & 3.90 \\
\hline $\begin{array}{l}\text { Average annual cyclists' crash frequency } \\
\text { at each intersection (crashes/intersection) }\end{array}$ & 1.23 & 1.57 \\
\hline $\begin{array}{l}\text { Average annual daily left turn traffic } \\
\text { (number of vehicles) }\end{array}$ & $11,869.73$ & $5,180.01$ \\
\hline $\begin{array}{l}\text { Average ratio of major AADT to minor } \\
\text { AADT }\end{array}$ & 2.03 & 2.12 \\
\hline $\begin{array}{l}\text { Average number of left turn lanes } \\
\text { (number of lanes) }\end{array}$ & 1.90 & 0.83 \\
\hline $\begin{array}{l}\text { Average number of through traffic lanes } \\
\text { (number of lanes) }\end{array}$ & 0.64 & 0.50 \\
\hline $\begin{array}{l}\text { Percentage of intersections with } \\
\text { push-button device (\%) }\end{array}$ & & 58.3 \\
\hline $\begin{array}{l}\text { Percentage of intersections with } \\
\text { countdown timer }(\%)\end{array}$ & & 43.7 \\
\hline
\end{tabular}

\section{Estimation Results}

The parameter estimation results and the corresponding average marginal effects are shown in Tables 2 and 3 . Table 2 that shows random parameters negative binomial model estimation indicates 4 significant fixed parameters, including 3 variables (annual average daily left turn traffic, ratio of major direction AADT to minor direction AADT, and number of through traffic lanes) and a constant, while there are 3 significant random parameters (push-button availability, countdown timer availability, and number of left turn lanes). Talking about each variable, it has been found that existence of push-button at intersection will significantly reduce average annual number of pedestrians' crashes. This is, obviously, expected given that pedestrians can use pushbutton and somehow push traffic signal to turn to green. The marginal effects in Table 2 show that a 1-unit increase in push-button could decrease the mean number of pedestrians' crashes per year at intersections by 4.03 . Similarly, availability of countdown timer has also positive impact on pedestrians' crashes reduction. Furthermore, as expected, increases in the left turn traffic volume, ratio of major direction AADT to minor direction AADT, and number of left turn and through traffic lanes increase the frequency of pedestrians' crashes at intersections. Although marginal effects are small, however still they are tangible and need to be counted in planning and operational levels. As these results indicate, any increase in the mentioned variables (traffic volume and number of lanes) can adversely affect safety of crossing pedestrians. The log-likelihood ratio index $\rho^{2}$ is 0.35 , indicating that proposed model could explain relationship between number of pedestrian crashes and selected variables sufficiently.

Turning to impacts of different variables on cyclists' crash frequency, almost the same trend as was obtained in case of pedestrians was observed. The estimated parameter for the average annual left turn traffic was found to be with

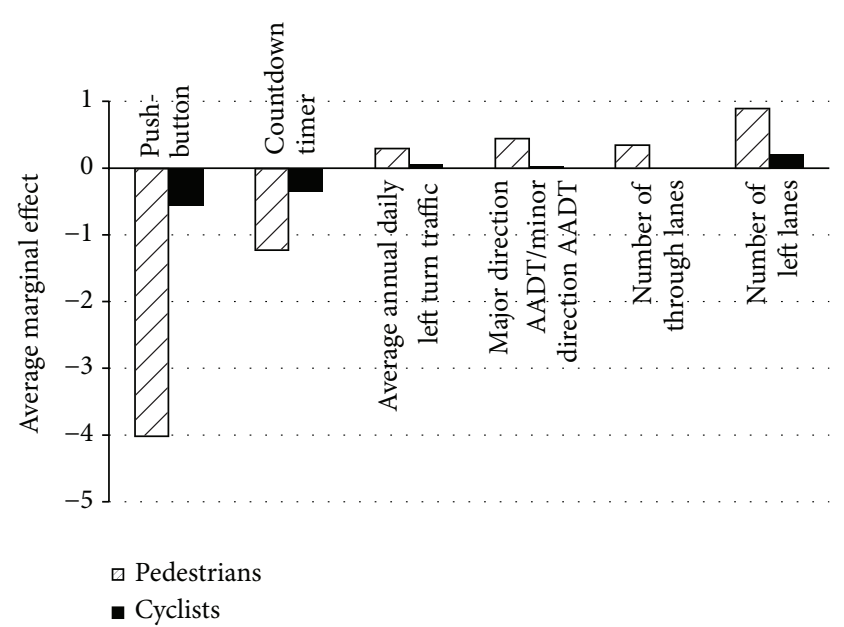

Figure 1: Average marginal effects of different parameters on pedestrians' and cyclists' crash frequencies.

a slightly negative but insignificant effect on average cyclists' crash frequency at intersections. This might be due to, among other factors, the fact that local drivers are more cautious for cyclists while making left turns. Additionally, it can also be interpreted in another way, that cyclists can cross the road faster than pedestrians and do not get involved in a crash due to left turn traffic. However, this statement does not hold for through traffic which has significant effect on cyclists' crash frequency. Based on the average marginal effects, cyclists are less sensitive to selected parameters compared to pedestrians. This is shown in Figure 1. Again, the log-likelihood ratio index $\rho^{2}$ was found to be 0.31 , indicating that the model has adequate explanatory and predictive power.

\section{Summary and Conclusions}

This study provides an empirical evaluation of the safety impacts of push-button and countdown timer on frequency of pedestrians' and cyclists' crashes at intersections within an urban network. Several variables including number of left turn and through traffic lanes, average annual daily left turn as well as through traffics on major and minor directions at intersection, existence of push-button, and countdown timer at each intersection have been considered for modeling. A random parameters negative binomial model was utilized to establish the statistical relationship between pedestrians'/cyclists' crash frequency and selected variables. The proposed model could overcome the overdispersion (mean less than variance) in the crash data, which is a general situation for traffic accident data analysis.

The estimation results show push-button and countdown timer unambiguously decrease the frequency of pedestrians' and cyclists' crashes. However, based on average marginal effect values, their effects are more significant and tangible on pedestrians' crash frequency, compared to crash frequency of cyclists. Number of left turn and through traffic lanes have negative impacts on both pedestrians' and cyclists' crash frequencies, although they produced higher $t$-stat values in 
TABLE 2: Model estimation results for random parameters negative binomial model of pedestrians' crash frequency at intersections (all random parameters are normally distributed).

\begin{tabular}{|c|c|c|c|}
\hline Variable & Parameter coefficient estimate & $t$-stat $(90 \% \mathrm{CI})$ & Average marginal effect \\
\hline Constant & 1.33 & 16.28 & \\
\hline $\begin{array}{l}\text { Push-button ( } 1 \text { if intersection has push-button; } 0 \\
\text { otherwise) (standard deviation of parameter } \\
\text { distribution) }\end{array}$ & $-0.81(-0.34)$ & $-10.03(-3.69)$ & -4.03 \\
\hline $\begin{array}{l}\text { Countdown timer ( } 1 \text { if intersection has countdown } \\
\text { timer; } 0 \text { otherwise) (standard deviation of parameter } \\
\text { distribution) }\end{array}$ & $-0.25(-0.87)$ & $-4.09(-12.82)$ & -1.24 \\
\hline Average annual daily left turn traffic (vehicle per day) & 0.06 & 1.71 & 0.30 \\
\hline $\begin{array}{l}\text { Ratio of major direction AADT to minor direction } \\
\text { AADT }\end{array}$ & 0.09 & 2.69 & 0.45 \\
\hline Number of through traffic lanes & 0.07 & 2.45 & 0.35 \\
\hline $\begin{array}{l}\text { Number of left turn traffic lanes (standard deviation of } \\
\text { parameter distribution) }\end{array}$ & $0.18(1.49)$ & $4.29(27.64)$ & 0.90 \\
\hline Dispersion parameter, $\alpha$ & 0.32 & 13.45 & \\
\hline Number of observations & & 1001 & \\
\hline Log-likelihood with constant only, LL(0) & & -2850.78 & \\
\hline Log-likelihood at convergence, $\operatorname{LL}(\boldsymbol{\beta})$ & & -2538.02 & \\
\hline$\rho^{2}=[1-\operatorname{LL}(\boldsymbol{\beta}) / \operatorname{LL}(0)]$ & & 0.35 & \\
\hline
\end{tabular}

TABLE 3: Model estimation results for random parameters negative binomial model of cyclists crash frequency at intersections (all random parameters are normally distributed).

\begin{tabular}{|c|c|c|c|}
\hline Variable & Parameter coefficient estimate & $t$-stat $(90 \% \mathrm{CI})$ & Average marginal effect \\
\hline Constant & 0.07 & 5.50 & \\
\hline $\begin{array}{l}\text { Push-button ( } 1 \text { if intersection has push-button; } 0 \\
\text { otherwise) (standard deviation of parameter } \\
\text { distribution) }\end{array}$ & $-0.45(-0.43)$ & $-3.67(-3.57)$ & -0.56 \\
\hline $\begin{array}{l}\text { Countdown timer ( } 1 \text { if intersection has countdown } \\
\text { timer; } 0 \text { otherwise) (standard deviation of parameter } \\
\text { distribution) }\end{array}$ & $-0.28(-0.31)$ & $-3.23(-3.82)$ & -0.35 \\
\hline Average annual daily left turn traffic (vehicle per day) & 0.05 & 1.54 & 0.06 \\
\hline $\begin{array}{l}\text { Ratio of major direction AADT to minor direction } \\
\text { AADT }\end{array}$ & 0.03 & 1.91 & 0.04 \\
\hline Number of through traffic lanes & 0.01 & 1.69 & 0.01 \\
\hline $\begin{array}{l}\text { Number of left turn traffic lanes (standard deviation of } \\
\text { parameter distribution) }\end{array}$ & $0.17(0.23)$ & $2.11(4.28)$ & 0.21 \\
\hline Dispersion parameter, $\alpha$ & 0.70 & 8.76 & \\
\hline Number of observations & & 1001 & \\
\hline Log-likelihood with constant only, LL(0) & & -1629.23 & \\
\hline Log-likelihood at convergence, $\operatorname{LL}(\boldsymbol{\beta})$ & & -1123.03 & \\
\hline$\rho^{2}=[1-\operatorname{LL}(\boldsymbol{\beta}) / \operatorname{LL}(0)]$ & & 0.31 & \\
\hline
\end{tabular}

case of pedestrians than cyclists. Similarly, left turn and through traffic volumes, as expected, have also considerable effects on crash frequencies. The findings of current study could provide valuable insights for pedestrians' and cyclists' safety improvement projects in both planning and operational levels. The other variables, such as weather conditions, vehicle types, and land use conditions, could be considered in future research in order to make the analysis results more robust.

\section{Conflict of Interests}

The authors declare that there is no conflict of interests regarding the publication of this paper.

\section{Acknowledgments}

This paper was supported by the Special Fund for Basic Scientific Research of Central Colleges, Chang'an University (no. 
CHD2012TD005) and Natural Science Foundation of China (no. 51208052). The authors are grateful for the assistance of transportation agencies in the Chicago metropolitan area for data collection as part of methodology application.

\section{References}

[1] M. M. Nesheli, O. C. Puan, and A. M. Roshandeh, "Optimization of traffic signal coordination system on congestion: a case study," WSEAS Transactions on Advances in Engineering Education, vol. 7, no. 6, pp. 203-212, 2009.

[2] A. M. Roshandeh, M. M. Nesheli, and O. C. Puan, "Evaluation of traffic characteristics: a case study," International Journal of Recent Trends in Engineering, vol. 1, no. 6, pp. 62-68, 2009.

[3] A. M. Roshandeh, H. S. Levinson, Z. Li, H. Patel, and B. Zhou, "A new methodology for intersection signal timing optimization to simultaneously minimize vehicle and pedestrian delays," ASCE Journal of Transportation Engineering, vol. 140, no. 5, Article ID 04014009, 2014.

[4] World Health Organization, Global Status Report on Road Safety 2013, World Health Organization, 2014.

[5] National Highway Traffic Safety Administration, "Traffic Safety Facts 2011 Data," 2013.

[6] J. Pucher and L. Dijkstra, "Promoting safe walking and cycling to improve public health: lessons from the netherlands and germany ", The American Journal of Public Health, vol. 93, no. 9, pp. 1509-1516, 2003.

[7] N. Eluru, C. R. Bhat, and D. A. Hensher, "A mixed generalized ordered response model for examining pedestrian and bicyclist injury severity level in traffic crashes," Accident Analysis \& Prevention, vol. 40, no. 3, pp. 1033-1054, 2008.

[8] A. M. Roshandeh and M. A. Aghmasjed, "Comparative assessment of turning movements at intersection using arcady and picady," International Journal of Modelling and Simulation, vol. 31, no. 4, pp. 271-278, 2011.

[9] C. Siddiqui, M. Abdel-Aty, and K. Choi, "Macroscopic spatial analysis of pedestrian and bicycle crashes," Accident Analysis and Prevention, vol. 45, pp. 382-391, 2012.

[10] Y. Liu and Y. Tung, "Risk analysis of pedestrians road-crossing decisions: effects of age, time gap, time of day, and vehicle speed," Safety Science, vol. 63, pp. 77-82, 2014.

[11] A. Abvabi, A. Nasr, A. Noorpoor, and M. S. Kiasat, "Investigation on the effect of impact location height on pedestrian safety using a legform impactor dynamic model," Safety Science, vol. 48, no. 5, pp. 660-671, 2010.

[12] D. J. Torbic, D. W. Harwood, C. D. Bokenkroger et al., "Pedestrian safety prediction methodology for urban signalized intersections," in Proceedings of the 89th Annual Meeting on Transportation Research Board, Compendium of Papers DVD, Washington, DC, USA, 2010.

[13] C. Hamann and C. Peek-Asa, "On-road bicycle facilities and bicycle crashes in Iowa, 2007-2010," Accident Analysis \& Prevention, vol. 56, pp. 103-109, 2013.

[14] D. N. Moore, W. H. Schneider IV, P. T. Savolainen, and M. Farzaneh, "Mixed logit analysis of bicyclist injury severity resulting from motor vehicle crashes at intersection and nonintersection locations," Accident Analysis and Prevention, vol. 43, no. 3, pp. 621-630, 2011.

[15] B. R. D. K. Agbelie and A. M. Roshandeh, "Impacts of signalrelated characteristics on crash frequency at urban signalized intersections," Journal of Transportation Safety \& Security, 2014.
[16] M. R. Ibrahim, M. R. Karim, and F. A. Kidwai, "The effect of digital count-down display on signalized junction performance," American Journal of Applied Sciences, vol. 5, no. 5, pp. 479-482, 2008.

[17] R. A. Tegge, J. H. Jo, and Y. Ouyang, "Development and application of safety performance functions for Illinois," Urbana, vol. 51, p. 61801, 2010.

[18] S. P. Washington, M. G. Karlaftis, and F. L. Mannering, Statistical and Econometric Methods for Transportation Data Analysis, CRC Press, Boca Raton, Fla, USA, 2nd edition, 2011.

[19] D. Lord and F. Mannering, "The statistical analysis of crashfrequency data: a review and assessment of methodological alternatives," Transportation Research A: Policy and Practice, vol. 44, no. 5, pp. 291-305, 2010.

[20] W. Greene, Version 9.0. Econometric Software Inc., Plainview, Tex, USA, 2007.

[21] M. Ben-Akiva and S. R. Lerman, Discrete Choice AnalysisTheory and Applications to Travel Demand, MIT Press, Cambridge, Mass, USA, 1985. 


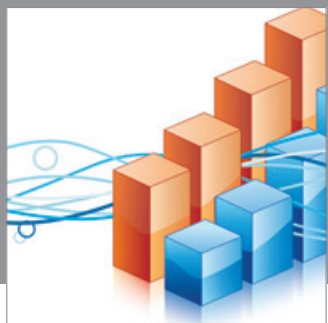

Advances in

Operations Research

mansans

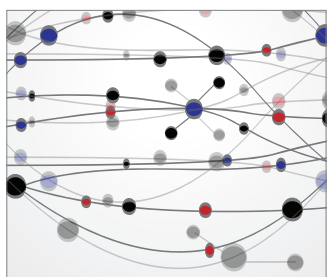

The Scientific World Journal
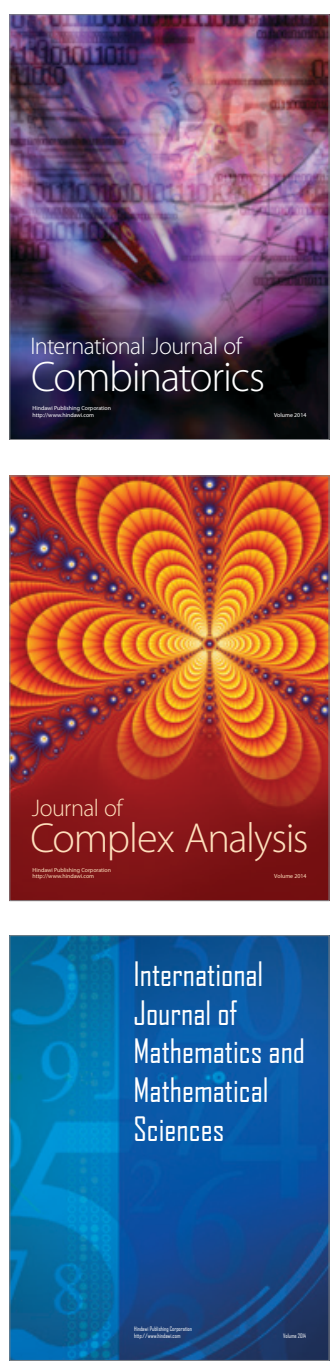
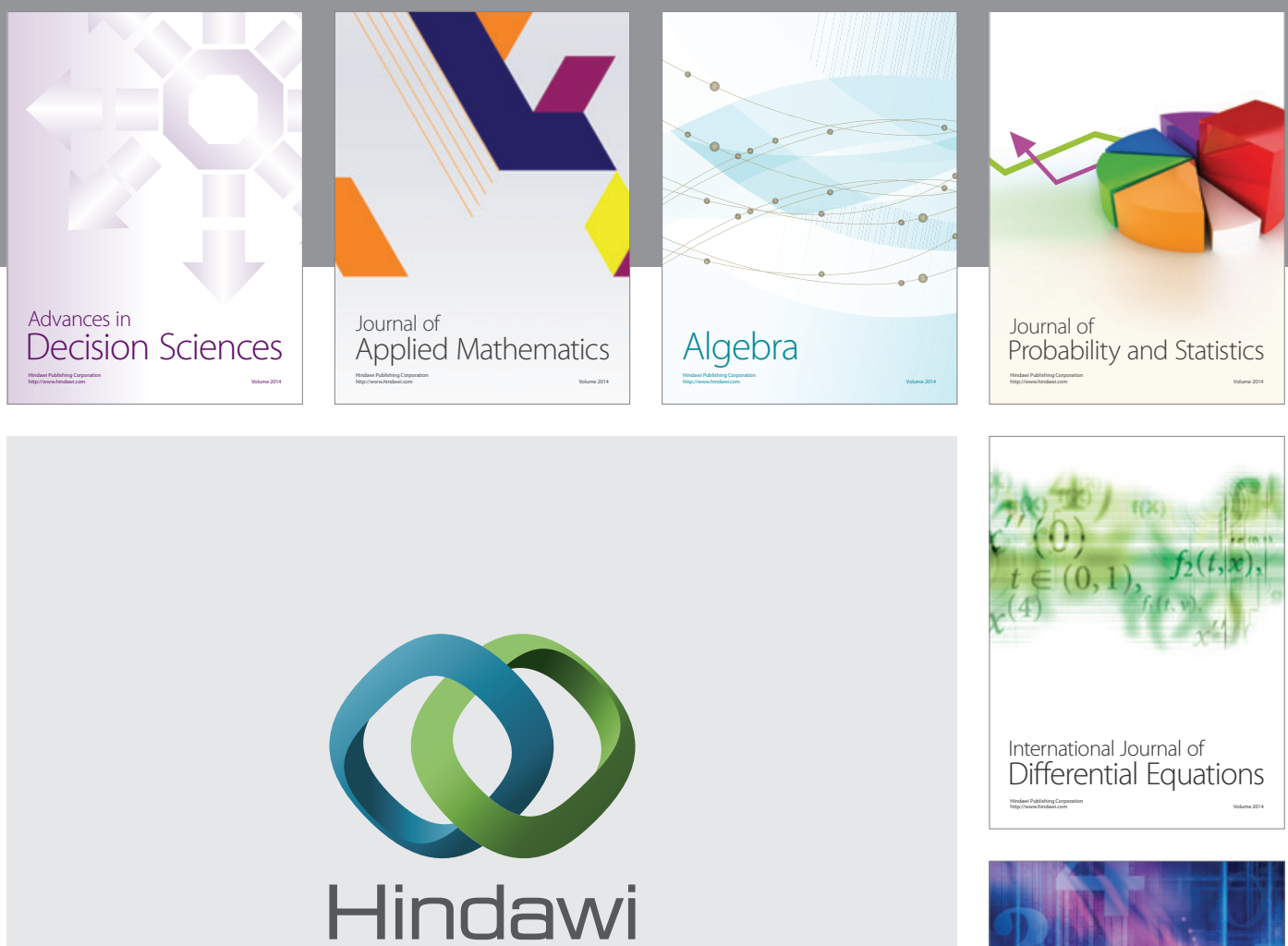

Submit your manuscripts at http://www.hindawi.com
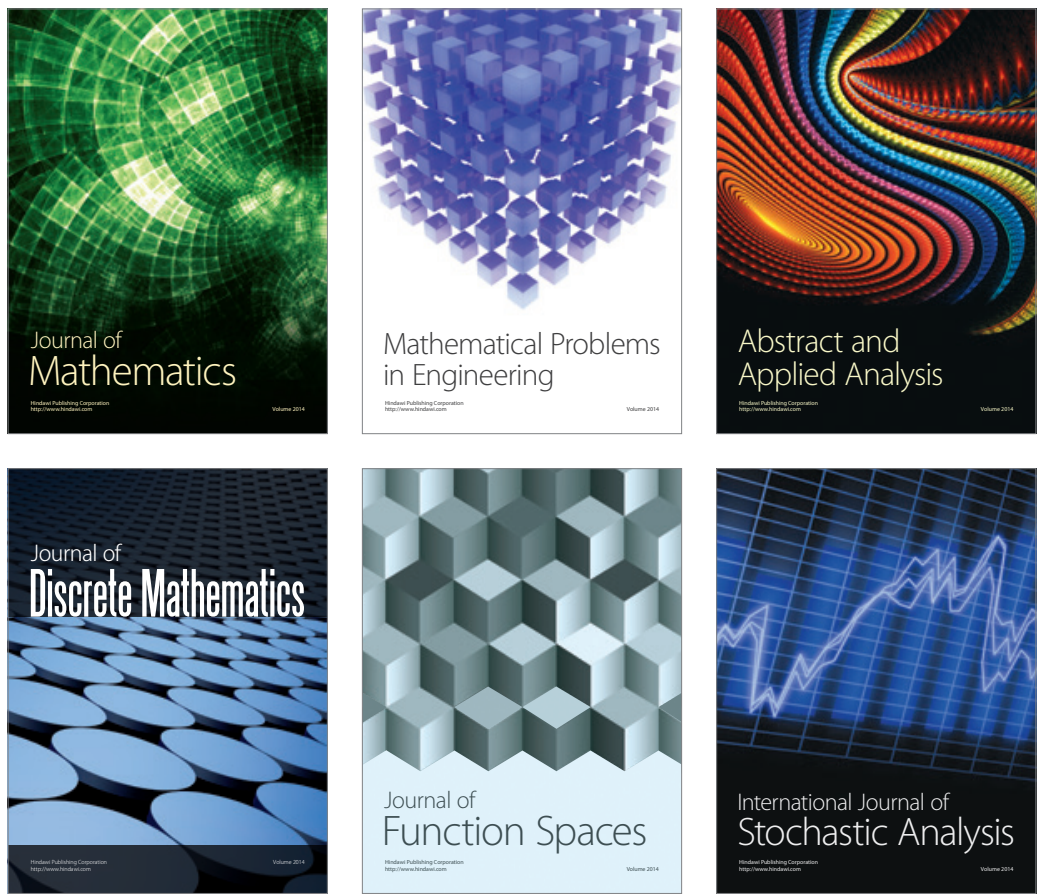

Journal of

Function Spaces

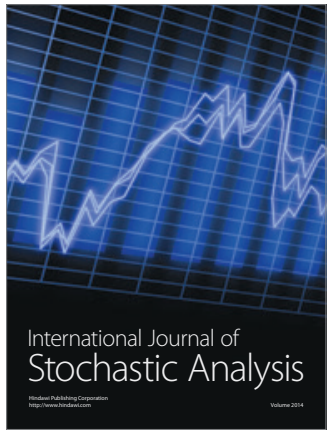

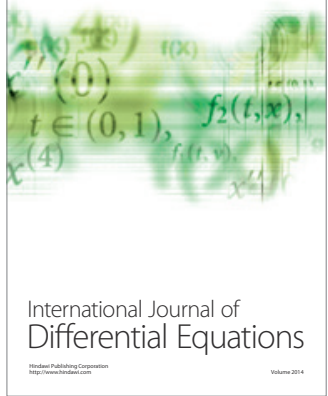
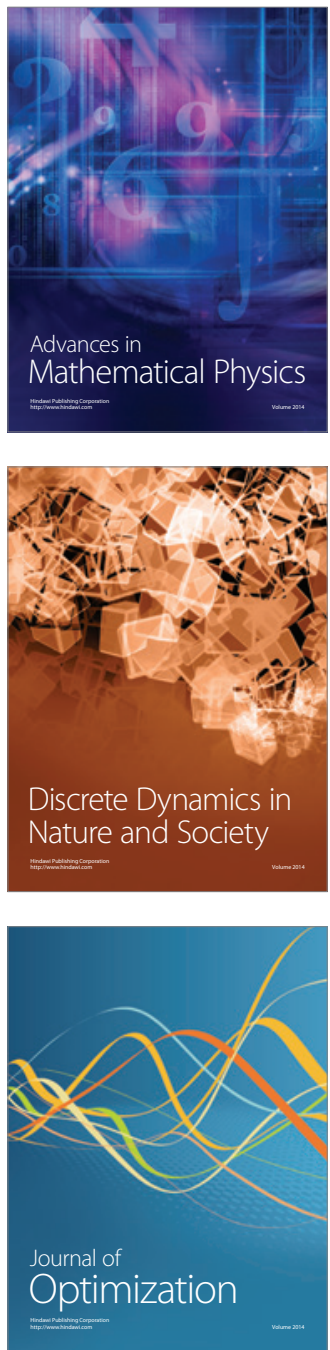